\title{
Escala de Insomnio de Atenas: evidencias psicométricas en adultos peruanos
}

\author{
Athens Insomnia Scale: Psychometric Evidence in Peruvian Adults
}

\author{
Jonatan Baños-Chaparro ${ }^{\mathrm{a}, *}$, Fiorella Gaby Fuster Guillen ${ }^{\mathrm{b}}$, Johnny Marín-Contreras ${ }^{\mathrm{c}}$ \\ aUniversidad Norbert Wiener, Perú \\ bUniversidad César Vallejo, Perú \\ cUniversidad de San Martín de Porres, Perú
}

\begin{abstract}
Resumen
Antecedentes: el insomnio es la alteración del sueño más frecuente en población adulta y en los últimos años se ha destacado como un importante problema de salud pública. La evidencia empírica sugiere que el insomnio se relaciona con problemas de salud mental y física. Por ello, resulta necesario identificar e intervenir este problema y disponer de instrumentos prácticos y confiables para su evaluación. Objetivos: analizar las propiedades psicométricas de la versión breve de la Escala de Insomnio de Atenas en adultos peruanos. Método: participaron 367 adultos peruanos seleccionados mediante muestreo por conveniencia y criterios de inclusión. Desde la Teoría Clásica de los Test (TCT) y la Teoría de Respuesta a los Ítems (TRI), se analizó las fuentes de evidencias de validez del instrumento. Resultados: el $65.9 \%$ indicó que tuvo dificultades para dormir durante el último mes. La escala evidencia una estructura unidimensional, aceptable confiabilidad, invarianza según el sexo y relación moderada con otra variable. Asimismo, los parámetros $a$ y $b$ fueron aceptables y la escala resulta ser más precisa en niveles altos de insomnio. Conclusiones: los hallazgos sugieren que la escala presenta adecuadas evidencias psicométricas y se recomienda su uso para evaluar el insomnio en población adulta peruana.
\end{abstract}

Palabras clave: insomnio; salud mental; adultos; propiedades psicométricas.

Baños-Chaparro, J., Fuster, F. G., \& Marín-Contreras, J. (2021). Escala de Insomnio de Atenas: evidencias psicométricas en adultos peruanos. Liberabit, 27(1), e458. https://doi.org/10.24265/ liberabit.2021.v27n1.07
Para citar este artículo:

\begin{abstract}
Background: Insomnia is the most frequent sleep disturbance in the adult population and, in recent years, it has been highlighted as an important public health problem. Empirical evidence suggests that insomnia is related to mental and physical health problems. Therefore, it is necessary to identify and intervene in this problem and have practical and reliable instruments for its evaluation. Objectives: To analyze the psychometric properties of the short version of the Athens insomnia scale in Peruvian adults. Method: Three hundred sixty-seven (367) Peruvian adults selected by convenience sampling and inclusion criteria participated in the study. Based on the classical test theory (CTT) and item response theory (IRT), the sources of validity evidence of the instrument were analyzed. Results: Sixty-five point nine percent (65.9\%) of the study population indicated that they had difficulty sleeping during the last month. The scale evidences a one-dimensional structure, acceptable reliability, invariance according to sex and moderate relationship with another variable. Likewise, parameters $a$ and $b$ were acceptable, and the resulting scale was more accurate at high levels of insomnia. Conclusions: The findings suggest that the scale presents adequate psychometric evidence, and its use is recommended to evaluate insomnia in the Peruvian adult population.
\end{abstract}

Keywords: Insomnia; mental health; adults; psychometrics properties.

Este es un artículo Open Access publicado bajo la licencia Creative Commons Atribución 4.0 Internacional. (CC-BY 4.0)

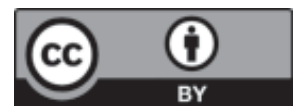

Universidad de San Martín de Porres, Lima - Perú

*banos.jhc@gmail.com 


\section{Introducción}

El insomnio es la alteración del sueño más frecuente en población adulta y afecta aproximadamente al 10-30\% de la población mundial (Ohayon, 2002). Un estudio multicéntrico reveló que los problemas de inducción al sueño eran más prevalentes en Japón (69\%) a comparación de Estados Unidos (57\%) y Europa Occidental (55\%); aunque los problemas para mantener el sueño fueron más frecuentes en Estados Unidos (78\%) que en Europa Occidental (75\%) o Japón (63\%) (Léger et al., 2008). En el Perú, un estudio epidemiológico de salud mental en Lima Metropolitana indicó que el $10.4 \%$ de los adultos calificaron su calidad de sueño como malo o bastante malo, mientras que el 32.3\% manifestó problemas de sueño que ameritaron atención médica. Además, el 30.6\% señaló despertarse durante la noche como problema principal para dormir (Instituto Nacional de Salud Mental [INSM], 2013). Por otro lado, en población clínica la prevalencia es aún mayor, donde se identificó que el $73.5 \%$ de los pacientes de un hospital público de Lima Metropolitana presentaron problemas de insomnio, siendo más frecuente en pacientes con hipertensión arterial (53.1\%) y diabetes (40.5\%) (Coloma et al., 2020).

Las personas que experimentan insomnio generalmente informan sobre dificultades para iniciar o mantener el sueño, despertares durante la noche o antes de lo deseado, la duración y la calidad del sueño (Ohayon, 2002). Las recomendaciones de National Sleep Foundations (NSF) sugieren que la duración del sueño apropiada para adultos de 18 a 64 años debe ser entre 7 y 9 horas, mientras que 7 y 8 horas para los adultos mayores de 65 años en adelante (Hirshkowitz et al., 2015). No obstante, las investigaciones en adultos han demostrado que no se logra dormir la cantidad recomendada, por ejemplo, se evidenció que los adultos peruanos residentes en Lima Metropolitana presentaban una duración de sueño menor a 7 horas (INSM, 2013). El hecho de que se experimente problemas para dormir es alarmante dado que se ha relacionado con una variedad de resultados perjudiciales. Por ejemplo, la evidencia empírica sugiere que el insomnio en población adulta mayormente se encuentra relacionado con problemas de ansiedad y sintomatología depresiva (La et al., 2020), ideas suicidas, desesperanza y estrés (Palagini et al., 2021), consumo de cafeína, tabaco y diversos desórdenes físicos (Ali et al., 2019). Respecto al sexo, las investigaciones indican mayor prevalencia en las mujeres (Ali et al., 2019; La et al., 2020), aunque otro estudio señala que no existen diferencias en relación al sexo (Sierra et al., 2008).

Por otro lado, se ha identificado que las personas que presentan constantemente dificultades para dormir, generalmente, no reconocen padecer un problema de insomnio (Ohayon, 2002). Aquello complica la situación de la persona, puesto que la evidencia indica que existen mayores probabilidades de experimentar alteraciones del sueño inducidas por estrés, depresión y ansiedad (Zhong et al., 2015). Por ello, es de suma importancia identificar, evaluar y brindar tratamiento psicológico o farmacológico (previa evaluación médica) respecto al insomnio. Una herramienta complementaria en la práctica clínica, no clínica e investigación básica o experimental, son los instrumentos psicométricos que recogen una muestra de conducta de una persona de forma objetiva y estandarizada, donde a partir de las puntuaciones obtenidas podemos realizar inferencias basadas acerca de un constructo, por ejemplo, insomnio (Muñiz, 2018). En ese sentido, un instrumento que destaca por su brevedad y fácil interpretación es la Escala de Insomnio de Atenas (Soldatos et al., 2000), que fue desarrollado basado en los criterios diagnósticos de la Clasificación Internacional de las Enfermedades (CIE-10), donde los primeros cinco ítems corresponden al criterio A y los tres últimos ítems, al criterio C. Asimismo, los encuestados responden en función si han experimentado aquellas dificultades al menos tres veces por semana durante el último mes, el cual corresponde al criterio B del CIE-10. El estudio original presenta dos versiones: una versión compuesta por 8 ítems (diseñado para su uso en entornos clínicos) y una versión breve de 5 ítems (evaluación específica de la dificultad con la cantidad y la calidad del sueño), 
ambas versiones presentan una estructura de un solo factor.

Actualmente, la versión breve presenta fuentes de evidencia psicométrica en España (Gómez-Benito et al., 2011; Sierra et al., 2008), China (Chiang et al., 2009) y Japón (Enomoto et al., 2018). En el Perú, para la evaluación de los problemas de sueño, se dispone de instrumentos que presentan fuentes de evidencia de validez como el Índice de Calidad de Sueño de Pittsburgh (Zhong et al., 2015) que evalúa la calidad del sueño y la Escala de Somnolencia de Epworth (Rosales-Mayor et al., 2012) que evalúa la somnolencia diurna. Sin embargo, no se dispone de instrumentos que evalúen el insomnio. Por lo tanto, el propósito de la investigación fue realizar el análisis psicométrico de la versión breve de cinco ítems de la Escala de Insomnio de Atenas en adultos peruanos. Los estudios psicométricos previos basan sus análisis desde el enfoque de la Teoría Clásica de los Test (TCT), la que permite aproximaciones a la dimensionalidad del constructo, consistencia interna y relación con otras variables. Sin embargo, no existe evidencia desde el enfoque de la Teoría de Respuesta a los Ítems (TRI) que se basa en el análisis de modelos logísticos que describen los ítems en función de parámetros y que, además, estima la precisión (confiabilidad) de los ítems o test (Muñiz, 2018). Presentar ambos enfoques reforzaría la calidad psicométrica del instrumento y su utilidad. Por ello, se propuso desarrollar los siguientes objetivos: a) analizar la estructura factorial de la escala, b) evaluar la consistencia interna y de constructo, c) analizar la invarianza de medición según el sexo, d) presentar la evidencia de validez basada en la relación con otras variables y e) analizar las características de los ítems y su función de información.

\section{Método}

El propósito del estudio es presentar las fuentes de evidencias psicométricas del instrumento. En ese sentido, la investigación se adhiere a la categoría de estudios instrumentales (Ato et al., 2013).

\section{Participantes}

Participaron 367 adultos peruanos, seleccionado mediante muestreo por conveniencia. Los criterios de inclusión fueron los siguientes: a) ser mayor de 18 años, b) lugar de residencia en Lima y c) haber aceptado el consentimiento informado. Los que no cumplieron con estos criterios no fueron considerados en el estudio. En ese sentido, participaron 170 mujeres (46.3\%) y 197 hombres (53.7\%), en un rango de edad de 19 a 71 años $(M=38.46 ; D E=$ 12.36). Además, se realizaron preguntas adicionales, por ejemplo, respecto al estado civil, el $46.6 \%$ era soltero, $42.5 \%$ casados, $8.3 \%$ divorciados y solo el $1.6 \%$ indicó que era viudo. La mayoría tenía un trabajo fijo (46.9\%) o temporal (20.7\%), aunque algunos mencionaron estar desempleados (32.4\%). Por último, se realizó la siguiente pregunta: ¿Has tenido dificultades para dormir o permanecer dormido, durante el último mes?, donde el 65.9\% indicó que sí tuvo dificultades, mientras que el 34.1\% señaló que no tuvo ningún problema.

\section{Instrumentos}

Cuestionario de datos demográficos. Se diseñó un apartado para la recopilación de información demográfica de los participantes, donde se averiguó el sexo, edad, estado civil, situación laboral, lugar de residencia y una pregunta relacionada con el insomnio.

Escala de Insomnio de Atenas. Fue desarrollado por Soldatos et al. (2000) para evaluar el insomnio según los criterios del CIE-10. La escala tiene dos versiones, una de 8 ítems y otra de 5 ítems. En este estudio se utilizó la versión en español de 5 ítems (Gómez-Benito et al., 2011), donde aquellos ítems evalúan la inducción del sueño, despertares durante la noche, despertares antes de lo deseado, duración y calidad del sueño. El sistema de respuesta es de tipo Likert, el cual se encuentra en un rango de 0 (ausencia del problema) a 3 (problema serio) y la puntuación general de la escala varía de 0 a 15 puntos. Los encuestados responden en función si han experimentado aquellas dificultades al menos tres 
veces por semana durante el último mes. La versión en español presenta un adecuado coeficiente alfa de .79 y evidencia de validez basada en la relación con otras variables, específicamente, con la sintomatología depresiva $(r=.46)$, ansiedad $(r=.42)$ y angustia psicológica $(r=.44)$. Por otro lado, se realizó un estudio piloto con 20 adultos peruanos residentes en Lima Metropolitana con la finalidad de examinar la comprensión de los ítems en la población de estudio. Todos los participantes indicaron que no tuvieron dificultad para comprender los ítems, ni tampoco preguntas o sugerencias. Se adjunta la escala (ver Anexo).

Patient Health Questionnaire-9 (PHQ-9). Es un instrumento que evalúa la sintomatología depresiva durante las últimas dos semanas y está diseñado a partir de los criterios establecidos por el Manual Diagnóstico y Estadístico de los Trastornos Mentales (DSM IV). El PHQ-9 está conformado por nueve ítems y tiene cuatro opciones de respuesta ( 0 = nada; 1 = varios días; 2 = más de la mitad de los días; 3 = casi todos los días), la puntuación general se encuentra en el rango de 0 a 27. En este estudio, se utilizó la validación en población peruana (Villarreal-Zegarra et al., 2019), la cual evidencia adecuada consistencia interna, reportando un coeficiente alfa de $.87 \mathrm{y}$ omega de .87. Asimismo, presenta invarianza de medición de acuerdo al sexo, edad, nivel educativo, nivel socioeconómico, estado civil y lugar de residencia.

\section{Procedimiento}

La recolección de los datos se realizó durante las dos últimas semanas de enero y primera semana de febrero de 2021. Debido a que en el Perú aún se mantiene el estado de emergencia sanitaria y las medidas de restricción para contrarrestar la COVID19, se elaboró la encuesta en línea mediante un formulario de Google. El enlace fue compartido a través de las redes sociales (Facebook y WhatsApp). El contenido del enlace indicaba el objetivo de la investigación, la confidencialidad de las respuestas, la utilidad de la información para fines académicos y su anonimato. Asimismo, al final de tal descripción se les preguntaba si deseaban participar voluntariamente en el estudio brindando su consentimiento. Si aceptaba participar, accedía a las preguntas de la encuesta, por el contrario, si no aceptaba, se daba por culminada su participación. Este estudio respetó la ética que rige la investigación en seres humanos, como el código de Nuremberg y la Declaración de Helsinki.

\section{Análisis de datos}

En principio, se realizó el análisis descriptivo de los ítems, considerando la media, la desviación estándar, la asimetría, la curtosis y la correlación ítem-test corregida, donde se espera valores superiores a .20 (Kline, 1986), también se reportó la matriz de correlación policóricas. Por otro lado, mediante el modelamiento de ecuaciones estructurales, se aplicó un análisis factorial confirmatorio (AFC) para evaluar la evidencia basada en la estructura interna. Se aplicó el estimador robusto de mínimos cuadrados ponderados ajustados a la media y la varianza (WLSMV, por sus siglas en inglés), el cual es recomendado para ítems ordinales (Kline, 2016). Para el ajuste del modelo se consideró el índice de ajuste comparativo (CFI), el índice de aproximación de la raíz de cuadrados medios del error (RMSEA) con IC del 90\%, la raíz estandarizada media cuadrática (SRMR) y la raíz cuadrada media residual ponderada (WRMR). Los valores del CFI deberán ser mayor a .95, el RMSEA menor a .06 y el SRMR menor a .08 (Hu \& Bentler, 1999; Kline, 2016); mientras que el WRMR deberá ser menor a 1 (DiStefano et al., 2018).

En relación a la consistencia interna, se realizó la estimación mediante el coeficiente omega (McDonald, 1999) y sus IC del 95\% en mil muestras Bootstrap bias-corregido y acelerado (BCa). No se aplicó el coeficiente alfa debido a que no se cumplió el supuesto de tau-equivalencia. Además, se analizó la confiabilidad de constructo mediante el coeficiente 
$\mathrm{H}$, cuyo propósito es evaluar si los ítems representan el rasgo latente, donde se espera valores superiores a .70 (Hancock \& Mueller, 2001).

La invarianza de medición se estableció para el grupo de hombres y mujeres. El análisis se realizó de manera gradual en diferentes condiciones, evaluando en principio la invarianza configural (sin restricciones), invarianza métrica (igualdad de cargas factoriales) e invarianza fuerte (igualdad de cargas factoriales e interceptos). El modelo se evalúo de acuerdo al CFI $>.95$ y el SRMR $<.08$ y para la comparación de las distintas condiciones, se consideró las diferencias del $\Delta \mathrm{CFI}<.01$ y $\Delta$ SRMR $<.03$ (Chen, 2007). Además, se analizó las diferencias de medias latentes entre los grupos si se cumple la condición de invarianza fuerte (Dimitrov, 2010).

Por último, para la evidencia basada en la relación con otras variables se aplicó la correlación de Pearson debido a que se cumplió con el supuesto de linealidad y variable de intervalo. Los cortes del tamaño del efecto para Pearson fueron los siguientes: .20 mínimo, .50 moderado y .80 fuerte (Ferguson, 2009). Asimismo, respecto al análisis basado en TRI, se aplicó el Modelo de Respuesta Graduada (GRM, por sus siglas en inglés), recomendado para ítems politómicos (Samejima, 1997). Debido a que la escala tiene cuatro opciones de respuesta, el modelo GRM estimó tres umbrales para cada ítem, describiendo el parámetro de discriminación (a) y parámetro de dificultad (b). Respecto al parámetro a, se siguió los criterios propuestos por Baker (2001), donde valores moderados se ubican entre .65 a 1.34 , valores altos entre 1.35 a 1.69 y valores muy altos mayores a 1.70. Adicionalmente, se analizó la función de información de la escala.

Los análisis estadísticos se realizaron en el software R versión 4.0.3 con el paquete lavaan (Rosseel, 2012), semPlot (Epskamp, 2015), MBESS (Kelley, 2019), psych (Revelle, 2018), statsExpression (Patil, 2020) y mirt (Chalmers, 2012).

\section{Resultados}

\section{Análisis descriptivo}

En la tabla 1 se evidencia que el rango de la media se encontró entre .84 y 1.09, es decir, entre el ítem 5 y el ítem 1. La desviación estándar más alta se encuentra en el ítem 1. Respecto a la asimetría y curtosis, todos los ítems se encuentran en \pm 1 .5. Por otro lado, la evaluación de la correlación ítem-test corregida demostró que todos los ítems fueron superiores al criterio de .20 (Kline, 1986), siendo el ítem 4 el que mayor discrimina. Asimismo, se evidencia en la matriz de correlación policóricas que no hubo presencia de multicolinealidad, es decir, intercorrelaciones mayores a .90 .

Tabla 1

Análisis descriptivo de los ítems

\begin{tabular}{|c|c|c|c|c|c|c|c|c|c|}
\hline Ítems & Media & $D E$ & $g_{1}$ & $g_{2}$ & $r_{\text {itc }}$ & \multicolumn{4}{|c|}{ Matriz de correlación policóricas } \\
\hline 1 & 1.09 & .76 & .27 & -.34 & .55 & & & & \\
\hline 2 & .96 & .74 & .14 & -.95 & .58 & .46 & & & \\
\hline 3 & 1.05 & .72 & .42 & .17 & .58 & .44 & .55 & & \\
\hline 4 & .93 & .71 & .38 & -.18 & .75 & .60 & .64 & .66 & \\
\hline 5 & .84 & .72 & .56 & .07 & 69 & .61 & .56 & .58 & .80 \\
\hline
\end{tabular}

Nota. $D E=$ desviación estándar, $g_{1}=$ asimetría, $g_{2}=$ curtosis, $r_{i t c}=$ correlación ítem test corregida. 


\section{Evidencia basada en la estructura interna}

El AFC con matrices policóricas y estimador robusto de WLSMV, demostró que el modelo unidimensional era aceptable con adecuados índices de ajuste: CFI = .99; RMSEA = .05 [IC 90\% = .00; $.09]$; $\mathrm{SRMR}=.02 ; \mathrm{WRMR}=.45 ; \mathrm{y}$ cargas factoriales $(\lambda)$ superiores a .50 (Figura 1). Asimismo, se evidenció ausencia de índices de modificación.

\section{Figura 1}

Estructura interna de la Escala de Insomnio de Atenas

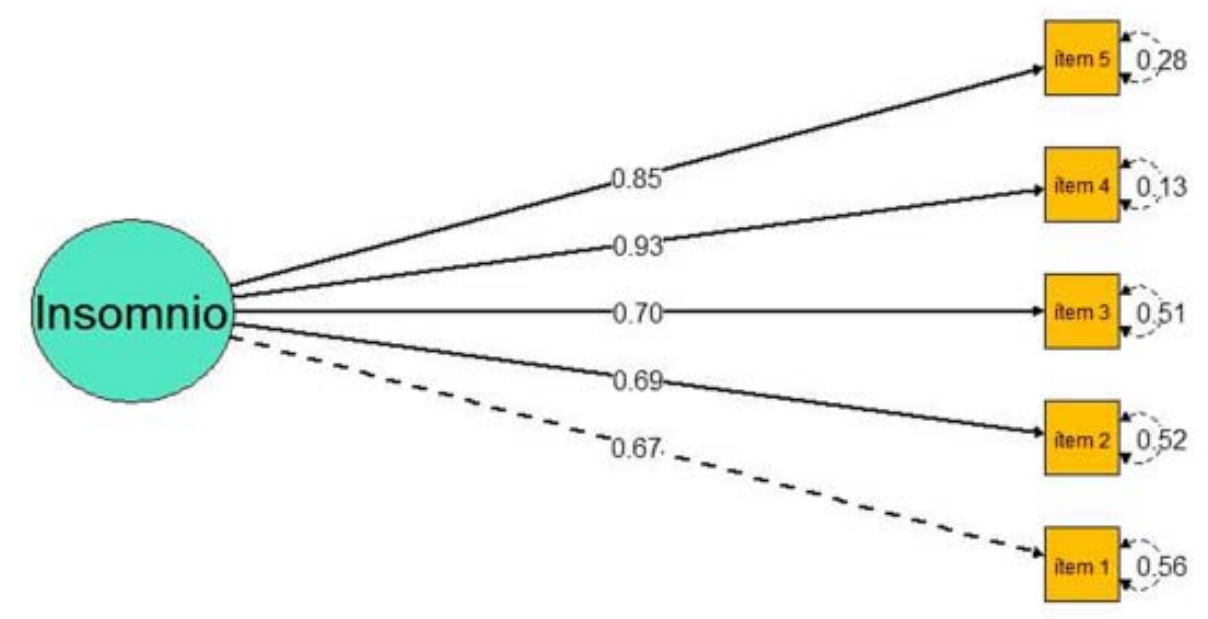

\section{Consistencia interna y constructo}

No se logró aplicar el coeficiente alfa debido a que no se cumplió el supuesto de tau-equivalencia CFI = .94; RMSEA = .21 [IC 90\% = .18; .23]; SRMR $=.09 ; \mathrm{y}$ WRMR $=2.14$. En ese sentido, se aplicó el coeficiente omega basado en mil muestras $\mathrm{BCa}$, el cual fue aceptable $\omega=.83$ [IC del $95 \%=$ .80; .86]. Además, se obtuvieron valores adecuados de confiabilidad de constructo $\mathrm{H}=.92$.

\section{Invarianza de medición}

El estimador robusto utilizado para evaluar las diferentes etapas de invarianza fue WLSMV. En ese sentido, se puede evidenciar en la tabla 2 que la variación entre una condición y otra fueron mínimas para el índice de ajuste comparativo $(\Delta \mathrm{CFI}<.01)$ y la raíz estandarizada media cuadrática $(\triangle \mathrm{SRMR}<$ .03). Por lo tanto, debido a que se logró cumplir la invarianza fuerte, se procedió a comparar las medias latentes donde no se encontró diferencias estadísticamente significativas en el sexo $\left(t_{(\mathrm{gl})}=\right.$ $\left..87_{(365)} ; p=.38\right)$.

Tabla 2

Invarianza de medición según sexo

\begin{tabular}{lcccccc}
\hline \multicolumn{1}{c}{ Grupos } & $\chi^{2}(g l)$ & $p$ & CFI & SRMR & $\Delta$ CFI & $\Delta$ SRMR \\
\hline Mujeres & $3.775(5)$ & .582 & 1.000 & .020 & & \\
Hombres & $17.620(5)$ & .003 & .989 & .043 & & \\
Configural & $23.238(10)$ & .009 & .999 & .032 & & \\
Métrica & $36.323(14)$ & .001 & .997 & .051 & .002 & .019 \\
Fuerte & $28.259(23)$ & .206 & 1.000 & .035 & .003 & .016 \\
\hline
\end{tabular}




\section{Evidencia basada en la relación con otras variables}

El análisis de correlación de las puntuaciones totales de la Escala de Insomnio de Atenas y el
PHQ-9 evidenció una relación positiva $(r=.68 ; p=$ .01) con un tamaño del efecto moderado (Figura 2).

\section{Figura 2}

Análisis de correlación del insomnio y la depresión

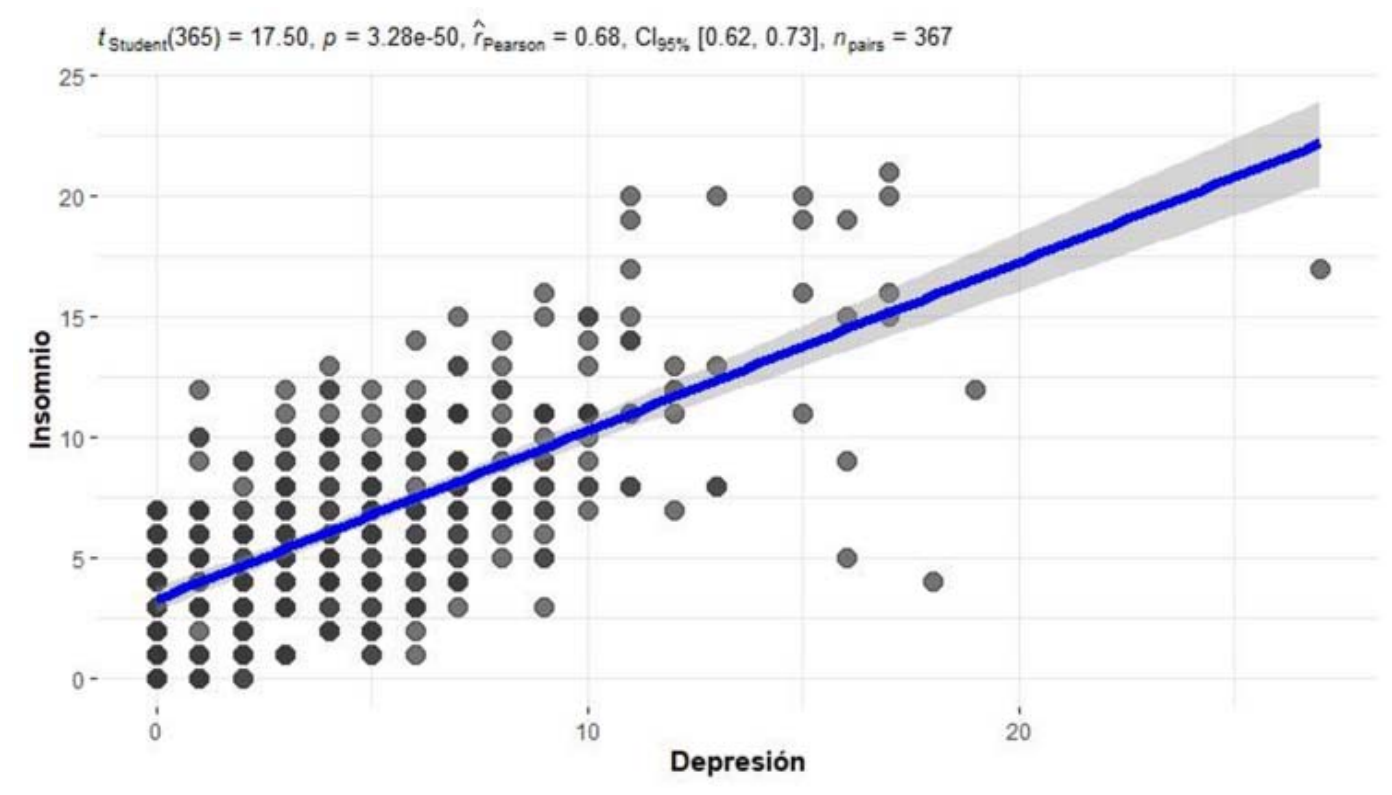

\section{Estimación y ajuste del modelo TRI}

Mediante el AFC se comprobó que los ítems destinados a medir la variable latente constituyen en una sola dimensión, lo cual confirma los supuestos de unidimensionalidad e independencia local de los ítems para el análisis de TRI. En ese sentido, el ajuste del modelo GRM fue aceptable: $\mathrm{CFI}=.99$; RMSEA = .04 [IC 95\% = .00; .09]; SRMR $=.02$. En la Tabla 3 , se evidencia los parámetros $a, b$ y sus errores estándar. En ese sentido, los parámetros de discriminación de todos los ítems fueron aceptables, evidenciando valores altos ( $>1.35)$ y muy altos ( $>$ 1.70 ), siendo el $a_{4}=4.91$ y el $a_{5}=3.15$ los mejores ítems que discriminan el rasgo. En relación al parámetro de dificultad, la mayoría fueron valores positivos y todos los umbrales aumentaron constantemente. Por otro lado, la función de información de la escala evidencia un valor máximo de 10.2 cuando el nivel de rasgo es $\theta=2.5$, lo que indica que el instrumento es más preciso en niveles altos de insomnio (Figura 3). 
Tabla 3

Parámetros de discriminación y dificultad para los ítems de la escala

\begin{tabular}{ccccccccc}
\hline Ítems & $a$ & ees & $b 1$ & ees & $b 2$ & ees & $b 3$ & ees \\
\hline 1 & 1.577 & .175 & -1.180 & .135 & .866 & .118 & 2.863 & .301 \\
2 & 1.727 & .191 & -.792 & .110 & .959 & .118 & 3.833 & .512 \\
3 & 1.823 & .198 & -1.144 & .124 & 1.040 & .117 & 2.657 & .259 \\
4 & 4.917 & .952 & -.618 & .076 & .937 & .084 & 2.259 & .178 \\
5 & 3.153 & .364 & -.487 & .080 & 1.168 & .100 & 2.371 & .199
\end{tabular}

Nota. $a$ = parámetro de discriminación, $b$ = parámetro de dificultad, ees = errores estándar.

\section{Figura 3}

Función de información de la Escala de Insomnio de Atenas

Test Information

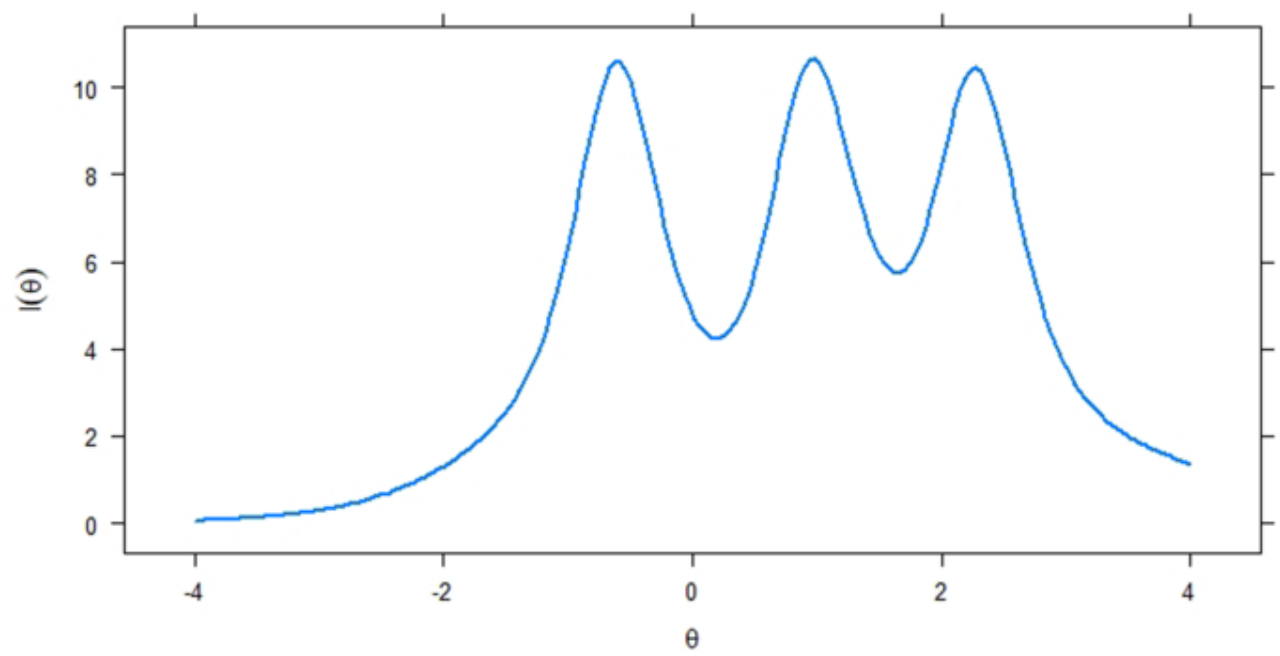

\section{Discusión}

En los últimos años, el insomnio se ha destacado como un importante problema de salud pública. La prevalencia de este problema ha sido identificada en diferentes países (Léger et al., 2008; Ohayon, 2002) $\mathrm{y}$, mayormente, se asocia con problemas de salud mental (La et al., 2020) y física (Ali et al., 2019). En el Perú, la población adulta considera que los problemas de sueño ameritaron atención médica y en población clínica la prevalencia supera el 70\% (Coloma et al., 2020; INSM, 2013). Esto conlleva a priorizar los problemas de insomnio en la atención primaria de salud para reducir los factores de riesgo asociados (Zhong et al., 2015) o las instituciones no vinculadas al sistema de salud, por ejemplo, universidades, colegios o empresas a brindar información necesaria sobre este problema y, por otra parte, contar con el apoyo de profesionales de la salud para la evaluación e intervención. Respecto a la evaluación, es necesario utilizar instrumentos confiables, prácticos y breves para una adecuada valoración. Por ello, el objetivo del estudio fue presentar las fuentes de evidencias de la versión breve de la Escala de Insomnio de Atenas en población adulta peruana.

En ese sentido, la evidencia basada en la estructura interna mediante el AFC, confirmó la estructura unidimensional del instrumento, 
presentando adecuados índices de ajuste, ausencia de índices de modificación y $\lambda$ superiores a .60. Estos resultados concuerdan con el estudio original donde también se evidenció una solución factorial de un factor y $\lambda$ superiores a .50 (Soldatos et al., 2000). También concuerda con los estudios de España (Gómez-Benito et al., 2011; Sierra et al., 2008), China (Chiang et al., 2009) y Japón (Enomoto et al., 2018), donde también se reportó una estructura unidimensional y $\lambda$ superiores a .60 .

La evaluación de la consistencia interna y de constructo mediante los coeficientes $\omega \mathrm{y} \mathrm{H}$ fueron aceptables, demostrando que los ítems son coherentes con el constructo de medición. Estos hallazgos concuerdan con las estimaciones de consistencia interna de otros estudios, que, aunque no aplicaron los coeficientes $\omega \circ \mathrm{H}$, presentan otro método alternativo para analizar la confiabilidad del instrumento como el coeficiente alfa $(\alpha)$, reportando valores de $\alpha$ entre .74 hasta .89 (Chiang et al., 2009; Enomoto et al., 2018; Gómez-Benito et al., 2011; Sierra et al., 2008; Soldatos et al., 2000). En este estudio no se logró aplicar el $\alpha$ porque no se logró cumplir con el supuesto de tau-equivalencia, donde se asume que las $\lambda$ son iguales en todos los ítems.

Respecto a la invarianza de medición, se desarrolló un modelo para la variable sexo, con el propósito de averiguar si el instrumento tiene las mismas propiedades de medición para los hombres y mujeres. En ese sentido, todas las etapas de medición gradual (configural, métrica y fuerte) presentaron adecuados índices de ajuste y aceptables valores de diferencias en las diversas etapas (Chen, 2007). Esto evidenció que el instrumento es equivalente para los hombres y mujeres. Por otro lado, debido a que se cumplió la etapa de invarianza fuerte (Dimitrov, 2010), se procedió a analizar si existen diferencias en relación al sexo, donde se evidenció que no hubo diferencias. Este resultado concuerda con la investigación de España, que también encontró ausencia de diferencias respecto al sexo (Sierra et al., 2008).
La evidencia basada en la relación con otras variables demostró que las puntuaciones de la Escala de Insomnio de Atenas se correlacionaron de manera positiva y estadísticamente significativa con el PHQ9 que evalúa la sintomatología depresiva. Estos resultados concuerdan con los estudios de validación de otros países, donde también se evidenció correlaciones positivas y estadísticamente significativas con la sintomatología depresiva (Enomoto et al., 2018; Gómez-Benito et al., 2011). Asimismo, estos hallazgos concuerdan con la literatura científica, la cual indica que las personas que experimentan problemas de insomnio, tienen mayores probabilidades de desarrollar desórdenes emocionales (Ali et al., 2019; Palagini et al., 2021; Zhong et al., 2015).

Por último, el ajuste del modelo TRI fue adecuado. En ese sentido, en relación al parámetro $a$, todos los ítems demostraron una potencia discriminativa entre alto y muy alto (Baker, 2001). Considerando que el parámetro $a$ refleja la representatividad del contenido del ítem vinculado al constructo, todos los ítems fueron adecuados para el rasgo latente. Por otro lado, en relación al parámetro $b$, la mayoría de los valores que se obtuvieron fueron positivos $\left(b_{2} \mathrm{y} b_{3}\right)$, a excepción del $b_{1}$ que presentó valores negativos. La predisposición de parámetros $b$ positivos demuestra que los ítems se encuentran localizados a un nivel medio y alto del rasgo latente (insomnio). Esto resulta ser coherente con el propósito de la escala, que fue construida en base a los criterios del CIE10 para detectar pacientes en riesgo (Soldatos et al., 2000). Asimismo, la función de información del test demostró que la información que aporta la escala es elevada en valores altos de insomnio, lo que indica que el instrumento es útil, confiable y preciso en individuos con niveles altos de insomnio.

Los hallazgos del estudio son alentadores y permiten brindar fuentes de evidencias de validez para la Escala de Insomnio de Atenas. Las implicancias del trabajo sugieren recomendar su uso en población adulta peruana para evaluar el insomnio y realizar 
comparaciones de acuerdo al sexo. Asimismo, se presentan evidencias de validez a nivel de los ítems, donde se han analizado las respuestas con los niveles del rasgo latente y la medición de su precisión, demostrando la calidad psicométrica del instrumento. La Escala de Insomnio de Atenas destaca por su brevedad y fácil interpretación. Estas características resultan importantes para su utilidad en estudios epidemiológicos de gran escala, herramienta de cribado en la atención primaria de salud y entre otros entornos no vinculados a un sistema de salud.

En esta misma línea, el estudio presenta algunas fortalezas, por ejemplo, los estudios psicométricos previos no presentan análisis de invarianza (Chiang et al., 2009; Enomoto et al., 2018; Gómez-Benito et al., 2011; Sierra et al., 2008); siendo este análisis necesario para realizar comparaciones de grupos (e.g., sexo) sin riesgo de sesgos. Además, tampoco presentan evidencias basadas desde el enfoque de TRI, lo cual fortalece los resultados de esta investigación, puesto que es el primer estudio en reportar evidencias basadas desde el enfoque TCT y TRI de la Escala de Insomnio de Atenas. No obstante, el estudio no está exento de limitaciones, por ejemplo, los resultados y conclusiones están basados en adultos residentes en Lima, lo cual no permite generalizar los resultados a otros departamentos del país y otras etapas de vida como la niñez o la adolescencia. En ese sentido, para mejorar los resultados se considera oportuno considerar otro tipo de población y realizar estudios confirmatorios en otros departamentos del país. Otra limitación del estudio es que solo se analizó la versión breve del instrumento, sugiriendo que los próximos estudios analicen la estructura interna de ocho ítems. Además, se sugiere considerar el análisis de curvas ROC para determinar la exactitud diagnóstica del test estableciendo un punto de corte con el objetivo de identificar a las personas en riesgo.

En conclusión, los hallazgos de la investigación sugieren que la Escala de Insomnio de Atenas es un instrumento que presenta fuentes de evidencias de validez adecuadas para las inferencias e interpretaciones de las puntuaciones en población adulta peruana, recomendando su uso para evaluar el insomnio y análisis comparativos de acuerdo al sexo.

\section{Conflicto de intereses}

Los autores declaran no tener ningún conflicto de interés económico, institucional, laboral o personal al realizar este artículo.

\section{Responsabilidad ética}

En el estudio no se realizó ningún procedimiento experimental. Durante el proceso de recolección de información se informó a cada participante el objetivo de la investigación, la confidencialidad de las respuestas, los fines académicos que conlleva la información recopilada y su participación anónima y voluntaria. Solo accedieron a la encuesta los participantes que aceptaron el consentimiento informado.

\section{Contribución de Autoría}

JBC: conceptualización y diseño del estudio, revisión bibliográfica, análisis estadísticos, redacción y aprobación final del manuscrito.

FGFG: revisión bibliográfica, recolección de datos, redacción y aprobación final del manuscrito.

JMC: revisión bibliográfica, recolección de datos, redacción y aprobación final del manuscrito.

\section{Referencias}

Ali, T., Belete, H., Awoke, T., Zewde, F., Derajew, H., Yimer, S., \& Menberu, M. (2019). Insomnia among Town Residents in Ethiopia: A Community-Based CrossSectional Survey. Sleep Disorders, 2019(8), 1-7. https:/ /doi.org/10.1155/2019/6306942

Ato, M., López, J. J., \& Benavente, A. (2013). Un sistema de clasificación de los diseños de investigación en 
Psicología. Anales de Psicología, 29(3), 1038-1059. http://doi.org/10.6018/analesps.29.3.178511

Baker, F. B. (2001). The Basics of Item Response Theory (2. ${ }^{\text {a }}$ ed.). ERIC Clearinghouse on Assessment and Evaluation.

Chalmers, R. P. (2012). mirt: A Multidimensional Item Response Theory Package for the R Environment. Journal of Statistical Software, 48(6), 1-29. https:// doi.org/10.18637/jss.v048.i06

Chen, F. F. (2007). Sensitivity of Goodness of Fit Indexes to lack of Measurement Invariance. Structural Equation Modeling: A Multidisciplinary Journal, 14(3), 464-504. https://doi.org/10.1080/10705510701301834

Chiang, H. L., Chen, H. C., Bai, C. H., Che, H. H., Lee, M. B., Lai, S. H., \& Chou, P. (2009). A Validation Study of the Chinese Version of the Athens Insomnia Scale. Taiwanese Journal of Psychiatry, 23(1), 43-52. https:/ /doi.org/10.29478/TJP.200903.0005

Coloma, F., Díaz, C., Espinoza, C., Flores, F., Godoy, G., Guelfand, S., Leyton, C., Mérida, F., \& Mora, M. (2020). Estudio de prevalencia de insomnio en 151 pacientes hospitalizados en servicio médico quirúrgico de un hospital público de la región metropolitana. Revista Confluencia, 2(1), 74-78. https://bit.ly/3b70osv

Dimitrov, D. M. (2010). Testing for Factorial Invariance in the Contex of Construct Validation. Measurement and Evaluation in Counseling and Development, 43(2), 121-149. https://doi.org/10.1177/0748175610373459

DiStefano, C., Liu, J., Jiang, N., \& Shi, D. (2018). Examination of the Weighted Root Mean Square Residual: Evidence for Trustworthiness? Structural Equation Modeling: A Multidisciplinary Journal, 25(3), 453-466. https:// doi.org/10.1080/10705511.2017.139

Enomoto, K., Adachi, T., Yamada, K., Inoue, D., Nakanishi, M., Nishigami, T., \& Shibata, M. (2018). Reliability and Validity of the Athens Insomnia Scale in Chronic Pain Patients. Journal of Pain Research, 11, 793-801. https:/ /doi.org/10.2147/JPR.S154852

Epskamp, S. (2015). semPlot: Unified Visualizations of Structural Equation Models. Structural Equation Modeling: A Multidisciplinary Journal, 22(3), 474483. https://doi.org/10.1080/10705511.2014.937847
Ferguson, C. J. (2009). An Effect Size Primer: A Guide for Clinicians and Researchers. Professional Psychology: Research and Practice, 40(5), 532-538. https://doi.org/ 10.1037/a0015808

Gómez-Benito, J., Ruiz, C., \& Guilera, G. (2011). A Spanish Version of the Athens Insomnia Scale. Quality of Life Research, 20(6), 931-937. https://doi.org/10.1007/ s11136-010-9827-x

Hancock, G. R., \& Mueller, R. O. (2001). Rethinking Construct Reliability within Latent Variable Systems. En R. Cudeck, S. H. Du Toit, \& D. Sörbom (Eds.), Structural Equation Modeling: Past and Present. A Festschrifi in Honor of Karl G. Jöreskoy (pp. 195-216). Scientific Software International.

Hirshkowitz, M., Whiton, K., Albert, S., Alessi, C., Bruni, O., DonCarlos, L., Hazen, N., Herman, J., Katz, E., Kheirandish-Gozal, L., Neubauer, D., O’Donnell, A., Ohayon, M., Peever, J., Rawding, R., Sachdeva, R., Setters, B., Vitiello, M., Ware, C., \& Adams, P. (2015). National Sleep Foundation's Sleep Time Duration Recommendations: Methodology and Results Summary. Sleep Health: Journal of the National Sleep Foundation, 1(1), 40-43. https://doi.org/10.1016/ j.sleh.2014.12.010

Hu, L. T., \& Bentler, P. M. (1999). Cutoff Criteria for Fit Indexes in covariance Structure Analysis: Conventional Criteria versus New Alternatives. Structural Equation Modeling: A Multidisciplinary Journal, 6(1), 1-55. https://doi.org/10.1080/10705519909540118

Instituto Nacional de Salud Mental - INSM. (2013). Estudio Epidemiológico de Salud Mental en Lima Metropolitana y Callao Replicación 2012. Informe general. Anales de Salud Mental, 29. https://bit.ly/3sFqXLg

Kelley, K. (2019). MBESS: The MBESS. R Package versión 4.6.0. https://bit.ly/3g7Dkcg

Kline, P. (1986). A Handbook of Test Construction: Introduction to Psychometric Design. Methuen.

Kline, R. B. (2016). Principles and Practice of Structural Equation Modeling (4.a ed.). The Guilford Press.

La, Y. K., Choi, Y. H., Chu, M. K., Nam, J. M., Choi, Y. C., \& Kim, W. J. (2020). Gender Differences Influence over Insomnia in Korean Population: A Cross-Sectional 
Study. PLoS ONE, 15(1), e0227190. https://doi.org/ 10.1371/journal.pone.0227190

Léger, D., Poursain, B., Neubauer, D., \& Uchiyama, M. (2008). An International Survey of Sleeping Problems in the General Population. Current Medical Research and Opinion, 24(1), 307-317. https://.doi.org/10.1185/ $030079907 X 253771$

McDonald, R. P. (1999). Test Theory: A Unified Treatment. Lawrence Erlbaum Associates, Inc.

Muñiz, J. (2018). Introducción a la psicometría. Teoría clásica y TRI. Madrid: Pirámide.

Ohayon, M. M. (2002). Epidemiology of Insomnia: What we Know and what we Still Need to Learn. Sleep Medicine Reviews, 6(2), 97-111. https://doi.org/10.1053/ smrv.2002.0186

Palagini, L., Miniati, M., Marazziti, D., Sharma, V., \& Rieman, D. (2021). Association Among Early Life Stress, Mood Features, Hopelessness and Suicidal Risk in Bipolar Disorder: The Potential Contribution of Insomnia Symptoms. Journal of Psychiatric Research, 135, 5259. https://doi.org/10.1016/j.jpsychires.2020.12.069

Patil, I. (2020). statsExpression: Tidy Dataframes and Expressions with Statistical Details. Journal of Open Source Software, 6(61), 3236, https://doi.org/10.21105/ joss.03236

Revelle, W. (2018). psych: Procedures for Personality and Psychological Research. https://bit.ly/3aKxgFp

Rosales-Mayor, E., Rey, J., Huayanay, L., \& Zagaceta, K. (2012). Validation and Modification of the Epworth Sleepiness Scale in Peruvian Population. Sleep and Breath, 16(1), 59-69. https://doi.org/10.1007/s11325011-0485-1
Rosseel, Y. (2012). lavaan: An R Package for Structural Equation Modeling. Journal of Statistical Software, 48(2), 36. https://doi.org/10.18637/jss.v048.i02

Samejima, F. (1997). Graded responde model. En W. J. Van der Linden, \& R. K. Hambleton (Eds.), Handbook of Modern Item Response Theory (pp. 85-100). Springer.

Sierra, J. C., Santos, P., \& Martínez, A. (2008). Análisis de la fiabilidad y validez de la versión reducida en español de la Athens Insomnia Scale (AIS-5). Cuadernos de Medicina Psicosomática y Psiquiatría de Enlace, 88, 11-16. https://bit.ly/37SafjT

Soldatos, C. R., Dikeos, D. G., \& Paparrigopoulos, T. J. (2000). Athens Insomnia Scale: Validation of an Instrument Based on ICD-10 Criteria. Journal of Psychosomatic Research, 48(6), 555-560. https:// doi.org/10.1016/s0022-3999(00)00095-7

Villarreal-Zegarra, D., Copez-Lonzoy, A., Bernabé-Ortiz, A., Melendez-Torres, G. J., \& Bazo-Alvarez, J. C. (2019). Valid Group Comparisons can be Made with the Patient Health Questionnaire (PHQ-9): A Measurement Invariance Study Across Groups by Demographic Characteristics. PLoS ONE, 14(9), e0221717. https:// doi.org/10.1371/journal.pone.0221717

Zhong, Q. Y., Gelaye, B., Sánchez, S. E., \& Williams, M. A. (2015). Psychometric Properties of the Pittsburgh Sleep Quality Index (PSQI) in a Cohort of Peruvian Pregnant Women. Journal of Clinical Sleep Medicine, 11(8), 869-877. https://doi.org/10.5664/jcsm.4936 


\section{Jonatan Baños-Chaparro}

Universidad Norbert Wiener, Perú.

Licenciado en Psicología clínica por la Universidad Norbert Wiener. Magister en educación con mención en docencia e investigación por la Universidad de San Martin de Porres. Formación en Terapia conductual cognitiva por el Centro Peruano de Investigaciones Psicológicas y Modificación del Comportamiento.

ORCID: https://orcid.org/0000-0002-2604-7822

Autor corresponsal: banos.jhc@gmail.com

Fiorella Gaby Fuster Guillen

Universidad César Vallejo, Perú.

Licenciada en Marketing y Dirección de empresas. Magister en educación con mención en docencia e investigación por la Universidad de San Martin de Porres. Formación en Gestión pública por la Pontificia universidad Católica del Perú y Evaluación de Impacto de Programas Sociales en Massachusetts Institute of Technology.

ORCID: https://orcid.org/0000-0003-3756-1541

fiore.gfg@gmail.com

Johnny Marín-Contreras

Universidad de San Martín de Porres, Perú.

Ingeniero de Computación y Sistemas por la Universidad de San Martín de Porres. Magister en educación con mención en docencia e investigación por la Universidad de San Martin de Porres. Gestión de Proyectos en la Universidad Católica de Chile. Certificación de Scrum Master.

ORCID: https://orcid.org/0000-0001-7275-1055

johnny_marin@hotmail.com 


\section{Anexo}

Escala de Insomnio de Atenas

1. Inducción del sueño (tiempo que tarda en dormirse después de apagar la luz)

0: Ningún problema

2: Marcadamente retrasado

2. Despertares durante la noche

0 : Ningún problema

1: Problema menor

2: Problema considerable

3. Despertar final antes de lo deseado
0 : No fue aantes
2: Notablemente antes

4. Duración total del sueño
0 : Suficiente
2: Notablemente insuficiente

1: Ligeramente insuficiente
1: Ligeramente retrasado

3: Muy retrasado o no durmió
3: Problema serio o no durmió
1: Un poco antes
3: Mucho antes o no durmió

5. Calidad general del sueño (no importa cuánto tiempo durmió)

0: Satisfactoria

1: Ligeramente insatisfactoria

2: Notablemente insatisfactoria

3: Muy insatisfactoria o no durmió

Nota. Adaptado de Gómez-Benito et al. (2011). 